\title{
A Robust Relay Selection Strategy for Cooperative Systems with Outdated CSI
}

\author{
Jose Lopez Vicario, Albert Bel, Antoni Morell, Gonzalo Seco-Granados \\ Group of Signal Processing for Communications and Navigation (SPCOMNAV) \\ Universitat Autonoma de Barcelona (UAB), 08193 Bellaterra (Barcelona), Spain \\ e-mail:\{jose.vicario, albert.bel, antoni.morell, gonzalo.seco\}@uab.es
}

\begin{abstract}
In this paper, we consider a cooperative system based on relay selection in a scenario where the available channel state information (CSI) is subject to delays. In order to exploit the selection diversity gains of the system while providing robustness against CSI inaccuracy, we propose a robust relay selection strategy based on a minimum mean square error (MMSE) Bayesian estimator. As shown in the paper, the proposed robust strategy provides significant gains in scenarios with different levels of CSI inaccuracy.
\end{abstract}

\section{INTRODUCTION}

In recent years much attention has been paid to the adoption of cooperative communications schemes [1][2]. This is mainly motivated by the fact that these schemes allow for the exploitation of spatial diversity gains without the need of multi-antenna technology. Indeed, most of the advantages of multiple-input multiple-output (MIMO) techniques can be extracted while keeping the complexity of the individual terminals reduced.

In terms of practical implementation, relay selection is a useful cooperative technique as it consists in only activating the best relay (in accordance to a given selection criterion) for retransmitting the source's information to the destination. Apart from the inherent simplicity of the proposed technique, this strategy avoids the need of synchronization (needed by most distributed space-time coding schemes) and reduces the power consumption of the terminals.

Concerning the selection criteria, outage optimality can be obtained by selecting the relay with the best instantaneous signal-to-noise ratio (SNR) [3]. When this selection strategy is implemented in a real system, however, the channel state of the selected relay at the selection decision instant can substantially differ from the actual channel during data transmission and, as a result, system performance is affected [4]. As an alternative, some works proposed a selection relaying mechanism based on localization knowledge [5][6]. A position-based scheme can outperform an opportunistic scheme with instantaneous information when channel state information (CSI) is not accurate but, however, the system is not able of exploiting the selection diversity gains provided by the cooperative system.

In this paper, we propose a relay selection strategy aimed at extracting the selection diversity gains of the system while providing robustness against CSI outdating. To do so, we adopt a Bayesian estimator based on the minimum mean square error (MMSE) criterion in order to estimate the actual SNR during data transmission from available measurements (outdated SNR

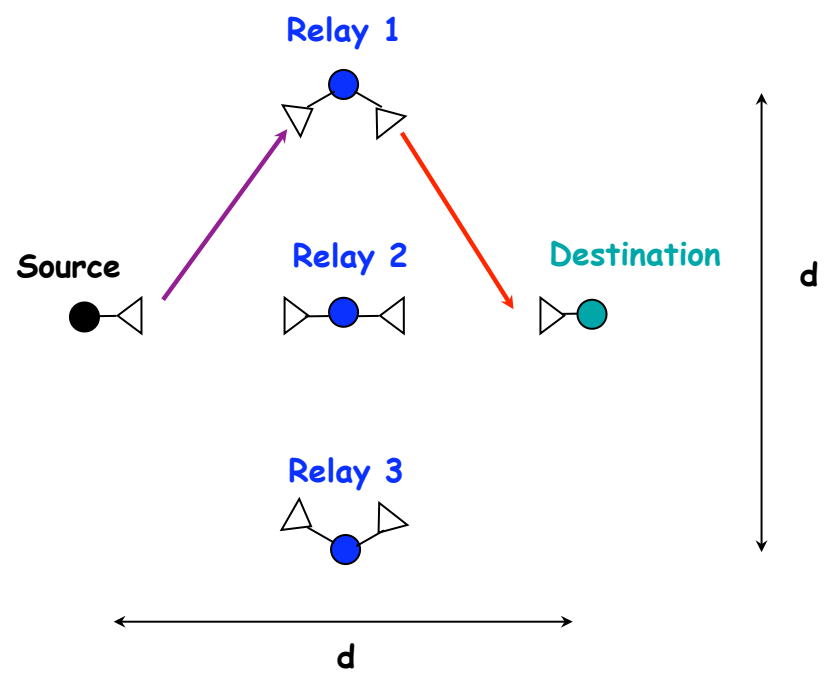

Fig. 1. Scheme of the proposed relaying strategy.

and position information). As shown in the paper, the proposed robust strategy provides signicant gains with respect to relay selection schemes based on either instantaneous SNR or localization information. Indeed, the proposed robust scheme provides the best performance for the whole range of CSI inaccuracy.

The rest of the paper is organized as follows. The corresponding system model is presented in Section II. In Section III, the proposed robust selection scheme is proposed. After that, performance of the proposed scheme is evaluated and compared with other relay selection schemes existing in the literature in Section IV. Finally, in Section V, the summary and conclusions of this paper are presented.

\section{System MODEL}

Consider a wireless network where one mobile unit (source) sends information to the base station (destination). In order to improve system performance, a cooperative mechanism is considered. In particular, a relay selection strategy is adopted in a scenario with $K$ mobile units of the network working as relays. In Fig. 1, we present an example of the proposed scenario, where relays are placed in a $d \times d$ square between the source and the destination, being $d$ also the distance of the source-destination link. 


\section{A. Signal Model}

For the sake of notation simplicity, we define an arbitrary link $A-B$ between two nodes $A$ and $B$. Node $A$ can be the source $(A=S)$ or the $k$-th relay $(A=k)$, while node $B$ can correspond to the $k$-th relay $(B=k)$ or to the destination $(B=D)$. With this model in mind, the received signal in the link $A-B$ can be written as:

$$
r_{B}=h_{A, B} x_{A}+n_{B}
$$

where $x_{A} \in \mathbb{C}$ is the transmitted symbol from node $A$ with power $P_{A}=\mathbb{E}\left[\left|x_{A}\right|^{2}\right], n_{B} \in \mathbb{C}$ is AWGN noise with zero mean and variance $\sigma_{n}^{2}, h_{A, B} \in \mathbb{C}$ is the channel response between nodes $A$ and $B$ modeled as $h_{A, B} \sim$ $C N\left(0, \sigma_{A, B}^{2}\right)$ (Rayleigh fading), being $\sigma_{A, B}^{2}$ the channel strength depending on the simplified path-loss model, $\sigma_{A, B}^{2}=$ $\left(\lambda_{c} / 4 \pi d_{o}\right)^{2}\left(d_{A, B} / d_{o}\right)^{-\mu}$, with $\lambda_{c}$ standing for the carrier wave-length, $d_{o}$ is a reference distance, $d_{A, B}$ is the distance of the link A-B and $\mu$ is the path-loss coefficient (being $\mu=3$ in this work). We consider a block-fading channel where the channel response remains constant during one time-slot and that the different channels are independently distributed. It is also assumed that each relay has an accurate estimate of its position (it is reasonable to assume that in the near future most terminals will be provided with positioning capabilities). Concerning power allocation, we consider that total transmit power of the system, $P$ is evenly distributed among the source and the selected relay, $k^{*}$, i.e. $P_{S}=P_{k^{*}}=0.5 P$. We denote by $\gamma_{A, B}=P_{A}\left|h_{A, B}\right|^{2} / \sigma_{n}^{2}$ the instantaneous signal-to-noise ratio (SNR) experienced in the link $A-B$ in a given time-slot and by $\bar{\gamma}_{A, B}=P_{A} \sigma_{A, B}^{2} / \sigma_{n}^{2}$ its long-term average.

\section{B. Relaying Mechanism}

Concerning the relaying procedure, we adopt a half-duplex two-hop decode and forward (DF) protocol as relaying strategy [2]. When using DF in a relay selection scheme, the subset of relays able to decode the information is named as the decoding subset $\mathcal{D S}$ and, from that subset, only the best relay (in accordance with the selection criterion) retransmits the information (see figure 2). As commented in Section III, system performance in a scenario with outdated CSI strongly depends on the selection criterion and, for that reason, this work focuses on the design of a robust selection scheme.

\section{Modeling of CSI Delay}

We consider that the SNR between relay $k$ and the destination available at the selection instant, $\hat{\gamma}_{k, D}$, can differ from the actual SNR $\gamma_{k, D}$ during information transmission due to channel variations. Indeed, we assume that $\hat{\gamma}_{k, D}$ are obtained from $\hat{h}_{k, D}$ (i.e., $\hat{\gamma}_{k, D}=P_{k}\left|\hat{h}_{k, D}\right|^{2} / \sigma_{n}^{2}$ ), which is an outdated version of $h_{k, D}$. Then, these two random variables are jointly Gaussian and, hence, $h_{k, D}$ conditioned on $\hat{h}_{k, D}$ follows a Gaussian distribution [7]:

$$
h_{k, D} \mid \hat{h}_{k, D} \sim \mathcal{C N}\left(\rho_{k} \hat{h}_{k, D},\left(1-\rho_{k}^{2}\right) \sigma_{k, D}^{2}\right)
$$

where parameter $\rho_{k}$ (with $0 \leq \rho_{k} \leq 1$ ) is the correlation coefficient between $\hat{h}_{k, D}$ and $h_{k, D}$ (degree of CSI accuracy),

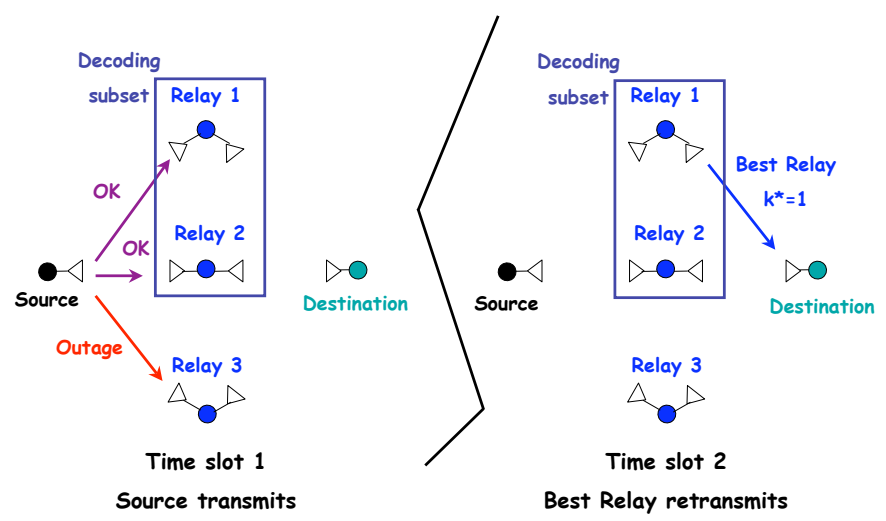

Fig. 2. Cooperative communications scheme based on relay selection and DF.

having different expressions according to the channel model. Under the assumption of a Jakes' model, for instance, the correlation coefficient takes the value $\rho_{k}=J_{o}\left(2 \pi f_{d_{k}} T_{D_{k}}\right)$, where $J_{o}(\cdot)$ denotes the zero-order Bessel function of the first kind, $f_{d_{k}}$ stands for the Doppler frequency and $T_{D_{k}}$ is time delay between the instants when the selection process is encompassed and the actual transmission of data from the selected relay takes place. Further details concerning the characteristics of $T_{D_{k}}$ can be found in [4].

Finally, it is worth mentioning that in this work we also assume that each relay has a perfect knowledge of parameter $\rho_{k}$. This is also a reasonable assumption taking into consideration that this parameter usually depends on Doppler frequency and $T_{D_{k}}$. Doppler frequency is usually estimated in digital receivers to carry out synchronization procedures and $T_{D_{k}}$ depends on the relaying protocol defined by the adopted communication standard.

\section{Performance Criterion}

In this work, performance of the cooperative system is measured in terms of the outage probability. The outage probability is defined as the probability that the instantaneous capacity of the system is below a predefined value $R$. In order to obtain the expression of the outage probability of the proposed two-hop DF scenario, one should start the analysis by studying the decoding subset $\mathcal{D S}$, i.e. the subset of relays able to decode the information transmitted by the source in the source-to-relay link:

$$
\mathcal{D S}=\left\{k: \log _{2}\left(1+\gamma_{S, k}\right) \geq 2 R\right\}=\left\{k: \gamma_{S, k} \geq 2^{2 R}-1\right\}
$$

Note that it is considered that relays are able to decode the source's information in the first hop when instantaneous capacity is higher than $2 R$, being this consideration also adopted in the relay-to-destination link. By doing so, the resulting end-toend spectral efficiency is $R$ as the proposed two-hop scheme requires two time-slots to transmit the information from the source to the destination.

For each combination of relays in the decoding set, the probability that the selected relay is not able to retransmit the 
source's information to the destination must be computed to obtain the outage probability of the system. By defining now $\mathcal{D} \mathcal{S}_{p}^{l}$ as the $p$-th element of the set of all possible decoding subsets with $l$ relays (i.e., $\mathcal{D S}_{p}^{l}$ is the $p$-th decoding subset of the $\left(\begin{array}{c}K \\ l\end{array}\right)$ possible subsets of $l$ relays taken from the $K$ relays), we can easily compute the outage probability as [3]:

$$
\operatorname{Prob}(\text { outage })=\sum_{l=0}^{K} \sum_{p=1}^{\left(\begin{array}{c}
K \\
l
\end{array}\right)} \operatorname{Prob}\left(\text { outage } \mid \mathcal{D S}_{p}^{l}\right) \operatorname{Prob}\left(\mathcal{D S}_{p}^{l}\right)
$$

where Prob(outage $\mid \mathcal{D} \mathcal{S}_{p}^{l}$ ) is the probability of outage conditioned on that the decoding subset is $\mathcal{D S}_{p}^{l}$ and $\operatorname{Prob}\left(\mathcal{D S} \mathcal{S}_{p}^{l}\right)$ is the probability of that subset.

\section{Robust Relay SELECTION}

As commented previously, the most popular selection strategies adopted in the literature are the following:

- Opportunistic Relay Selection (ORS): the relay maximizing the SNR in the link $k-D$ is selected, i.e.

$$
k_{O R S}^{*}=\arg \max _{k \in \mathcal{D} \mathcal{S}}\left\{\hat{\gamma}_{k, D}\right\}
$$

- Position-based Relay Selection (POS): in this case the relay closest to the destination is selected as follows

$$
k_{P O S}^{*}=\arg \min _{k \in \mathcal{D} \mathcal{S}}\left\{d_{k, D}\right\}
$$

where $d_{k, D}$ is the distance between relay $k$ and the destination.

As shown in [4], performance of ORS is substantially affected when CSI is not accurate. In those situations, it could be preferable to use the position-based approach (which is independent of CSI accuracy). However, selection diversity gains are not captured when CSI becomes more accurate.

Some of the conclusions above can be observed in Fig. 3, where a comparison between the ORS and POS based schemes is performed in terms of the outage probability (Eq. (1)). For the sake of benchmarking, results corresponding to a scenario without cooperation are included and system SNR is defined as the average received SNR of this single-hop scheme. As shown in the figure, the best performance is obtained with ORS when perfect CSI is assumed $(\rho=1)$. However, performance is quite sensitive to the value of $\rho$. When $\rho=0.3$, in particular, one can observe that only a slight improvement can be obtained by using ORS with respect to a non-cooperative scheme and that it is better to adopt the POS approach. Indeed, the behavior of the POS approach is independent of the value of $\rho$ but, as observed in the figure, this characteristic becomes a drawback when the value of $\rho$ increases.

In order to circumvent the problems commented above, we propose a robust scheme based on positioning information aimed at extracting the selection gain of ORS. Indeed, we propose a scheme where the selection of the active relay is performed by means of both the available CSI and the positioning information, that is to say:

$$
k_{R O B U S T}^{*}=f\left(\hat{\gamma}_{k, D}, d_{k, D}\right)
$$

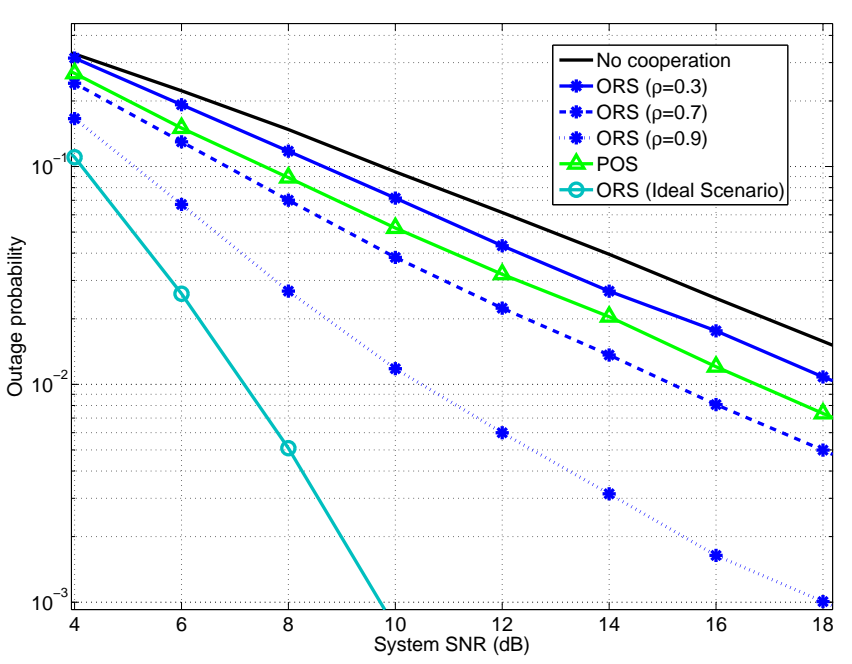

Fig. 3. Outage probability vs. system SNR for different selection strategies ( $K=5$ relays, $R=1 \mathrm{bit} / \mathrm{s} / \mathrm{Hz}$.).

To do so, we adopt a Bayesian estimator based on the MMSE criterion to estimate the actual SNR, $\gamma_{k, D}$, from parameters $\hat{\gamma}_{k, D}$ and $d_{k, D}$, being the estimate obtained as[8]:

$$
\hat{\hat{\gamma}}_{k, D}=\mathbb{E}\left(\gamma_{k, D} \mid \hat{\gamma}_{k, D}, d_{k, D}\right)
$$

Indeed, the proposed Bayesian estimator is adopted to select the relay with the highest SNR estimate. In other words, the proposed robust relay selection is that choosing the relay satisfying:

$$
\begin{aligned}
k_{R O B U S T}^{*} & =\arg \max _{k \in \mathcal{D} \mathcal{S}}\left\{\hat{\hat{\gamma}}_{k, D}\right\} \\
& =\arg \max _{k \in \mathcal{D} \mathcal{S}}\left\{\mathbb{E}\left(\gamma_{k, D} \mid \hat{\gamma}_{k, D}, d_{k, D}\right)\right\}
\end{aligned}
$$

In order to obtain a closed-form expression for the estimate $\hat{\hat{\gamma}}_{k, D}$, one should notice that in the proposed scenario there exists a one-to-one relation between the long-term average SNR, $\bar{\gamma}_{k, D}$, and $d_{k, D}$. Hence, we can rewrite the proposed estimator as:

$$
\hat{\hat{\gamma}}_{k, D}=\mathbb{E}\left(\gamma_{k, D} \mid \hat{\gamma}_{k, D}, \bar{\gamma}_{k, D}\right)
$$

By taking now into account the CSI uncertainty assumption, it is straightforward to show that the actual SNR, conditioned on its estimate, $\hat{\gamma}_{k, D}$ and its long-term average follows a non-central chi-square distribution with 2 degrees of freedom, whose probability density function (pdf) takes the following expression [7]:

$$
\begin{aligned}
f_{\gamma_{k, D} \mid \hat{\gamma}_{k, D}}\left(\gamma_{k, D} \mid \hat{\gamma}_{k, D}\right)=\frac{1}{\bar{\gamma}_{k, D}\left(1-\rho_{k}^{2}\right)} \\
\cdot e^{\frac{-\left(\gamma_{k, D}+\rho_{k}^{2} \hat{\gamma}_{k, D}\right)}{\bar{\gamma}_{k, D}\left(1-\rho_{k}^{2}\right)}} I_{0}\left(\frac{2 \sqrt{\rho_{k}^{2} \gamma_{k, D} \hat{\gamma}_{k, D}}}{\bar{\gamma}_{k, D}\left(1-\rho_{k}^{2}\right)}\right)
\end{aligned}
$$

where $I_{0}(\cdot)$ stands for the zero-order modified Bessel function of the first kind and one should take into consideration 


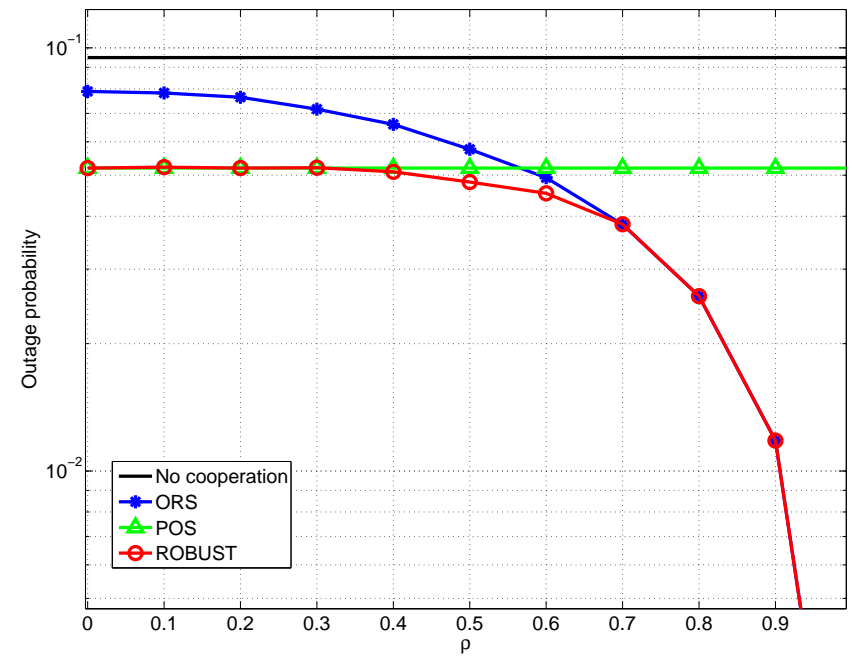

Fig. 4. Outage probability vs. $\rho$ parameter for different selection strategies ( $K=5$ relays, $R=1 \mathrm{bit} / \mathrm{s} / \mathrm{Hz}$, System $\mathrm{SNR}=10 \mathrm{~dB}$ ).

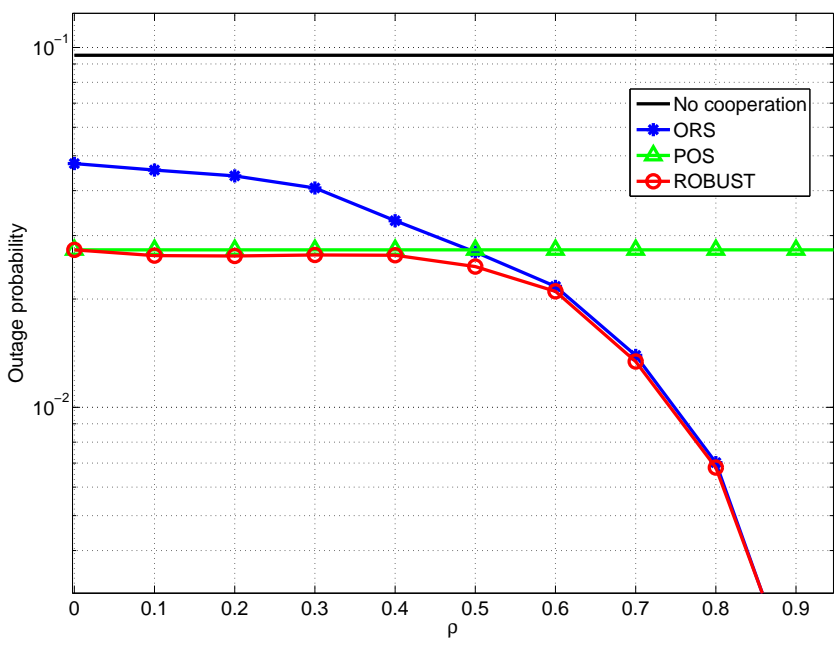

Fig. 5. Outage probability vs. $\rho$ parameter for different selection strategies ( $K=15$ relays, $R=1 \mathrm{bit} / \mathrm{s} / \mathrm{Hz}$, System $\mathrm{SNR}=10 \mathrm{~dB}$ ).

that the long-term average of $\hat{\gamma}_{k, D}$ is equal to $\mathbb{E}\left[\hat{\gamma}_{k, D}\right]=$ $\mathbb{E}\left[\left|\hat{h}_{k, D}\right|^{2}\right] P_{k} / \sigma_{n}^{2}=\mathbb{E}\left[\left|h_{k, D}\right|^{2}\right] P_{k} / \sigma_{n}^{2}=\bar{\gamma}_{k, D}$.

As a result, the problem of obtaining a robust estimate of the SNR is reduced to obtain the first moment of a non-central chi-square random variable with pdf given by (3). One can verify that this first moment can be expressed as [9]:

$$
\mathbb{E}\left(\gamma_{k, D} \mid \hat{\gamma}_{k, D}, \bar{\gamma}_{k, D}\right)=\rho_{k}^{2} \hat{\gamma}_{k, D}+\left(1-\rho_{k}^{2}\right) \bar{\gamma}_{k, D}
$$

Finally, by including the above result in (2), the selection rule of the proposed robust strategy is obtained as follows:

$$
\left.k_{R O B U S T}^{*}=\arg \max _{k \in \mathcal{D} \mathcal{S}}\left\{\rho_{k}^{2} \hat{\gamma}_{k, D}+\left(1-\rho_{k}^{2}\right) \bar{\gamma}_{k, D}\right)\right\}
$$

\section{NumericAl Results}

As far as numerical results are concerned, we consider the cooperative scenario represented in Fig. 1. In the proposed scenario, relays are randomly placed in the defined $d \times d$ square between the source and the destination.

We start the analysis by showing in Fig. 4 the outage probability of the different relay selection strategies as a function of the CSI uncertainty collected by parameter $\rho$ in a scenario with $K=5$ relays. Again, we also consider the baseline scheme without cooperation and we define system SNR as the average received SNR of this single-hop scheme. In the presented figure, one can observe how the choice of the position-based approach is appropriate in the low- $\rho$ region, whereas ORS pays off when the CSI becomes more accurate. Concerning the proposed robust strategy, it is able of extracting the robustness of the POS scheme and the capability of exploiting selection diversity gains of ORS. Indeed, the best performance can be obtained in the whole range by adopting the proposed robust criterion.

In Fig. 5, we present results related to a similar scenario where the number of relays is increased to $K=15$. In this case, performance of the cooperative mechanisms is substantially improved with respect to the non-cooperative case. This is because the benefits of using a relaying mechanism are more significant as having more available relays in the network increases the probability of selecting relays with better channel conditions. Besides, one can also observe that ORS performs better than POS for lower values of $\rho$. The reason for that being that the capability of generating SNR peaks of ORS is higher when the number of relays grows and, hence, compensates for SNR uncertainties. As a consequence, the robustness provided by POS is exploited in a narrower range of $\rho$. The proposed robust scheme, on the other hand, performs again better in the whole range of CSI uncertainties. Furthermore, benefits obtained in this particular case are more remarkable due to the increased selection gain resulting from considering a scenario with a higher number of relays.

Finally, it is worth noting that adopting a switching criterion between ORS and POS could be impractical as switching decisions depend on several parameters (such as position of the nodes, transmit and noise power, $\rho_{k}$ values, number of nodes) and a huge amount of situations must be considered to construct look-up tables. By adopting the proposed robust criterion, however, the best node is directly selected from available measurements.

\section{COnClusions}

In this paper we have addressed the problem observed when using a relay selection mechanism in a scenario with outdated CSI. In such a scenario, performance of ORS is substantially affected when CSI is not accurate. By using a positionbased approach, however, system performance is independent of CSI accuracy but selection gains are not captured when CSI becomes more accurate. In order to circumvent these problems, we have proposed a robust relay selection scheme able of capturing the selection gains of ORS while providing 
the robustness of POS. In particular, the proposed relay selection technique is based on a MMSE Bayesian estimator of the actual SNR during data transmission from available measurements (outdated SNR and position information). As proved in the paper, the proposed technique always performs better than its ORS and POS counterparts independent of the degree of CSI accuracy.

\section{ACKNOWLEDGMENT}

This work was supported by the Spanish Government Project TEC2008-06305/TEC.

\section{REFERENCES}

[1] J. N. Laneman, D. Tse, and G. W. Wornell, "Cooperative diversity in wireless networks: Efficient protocols and outage behaviour," IEEE Trans. On Information Theory, vol. 50, no. 12, pp. 3062-3080, Dec. 2004.
[2] R. U. Nabar, H. Bolcskei, and F. W. Kneubuler, "Fading relay channels: Performance limits and space-time signal design," IEEE JSAC, Aug. 2004.

[3] A. Bletsas, H. Shin, and M. Z. Win, "Cooperative communications with outage-optimal opportunistic relaying," IEEE Trans. on Wireless Comm., vol. 6, no. 9, Sept. 2007.

[4] A. Bel, J.L. Vicario, and G. Seco-Granados, "The benefits of relay selection in WiMAX networks," in Proc. ICT Mobile Summit, June 2008

[5] M. Zorzi and R. R. Rao, "Geographic random forwarding (GeRaF) for ad hoc and sensor networks: Multihop performance," IEEE Trans. Mobile Comp., vol. 2, pp. 337-348, Oct. 2003

[6] B. Zhao and M. C. Valent, "Practical relay networks: A generalization of hybrid-ARQ," IEEE Journ. on Sel. Areas in Comm., vol. 23, no. 1, pp. 7-18, Jan. 2005.

[7] J.L. Vicario and C. Antn-Haro, "Analytical assessment of multi-user vs. spatial diversity trade-offs with delayed channel state information," IEEE Comm. Letters, Aug. 2006.

[8] S. M. Kay, Fundamentals of Statistical Signal Processing: Estimation Theory, Vol. I, Prentice Hall, New Jersey, 1993.

[9] J.G. Proakis, Digital Communications, Mc Graw Hill, New York, 2001. 